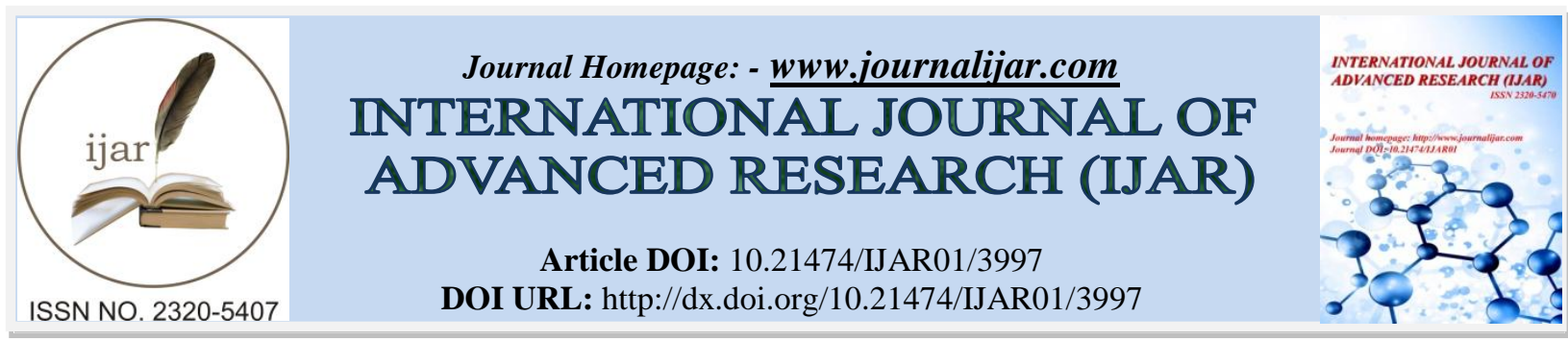

RESEARCH ARTICLE

\title{
FASUDIL HYDROCHLORIDE EFFECTS ON EAAT2 AND ROCK2 PROTEIN EXPRESSIONS IN RAT MODEL OF NEONATAL HYPOXIC-ISCHEMIC ENCEPHALOPATHY.
}

\author{
Ndefi A ntima Yadiswa Robert ${ }^{1}$, Wang Xian $\mathrm{He}^{2}$, Zhang Ya Li ${ }^{1}$, Zhu Zhang Long ${ }^{1}$, Raihan Kibria ${ }^{4}$, Nalika \\ Robai $^{3}$ and Indranil Ghosh ${ }^{3}$. \\ 1. Department of pediatrics unit 1,First Affiliated Hospital of Jiamusi University. \\ 2. Professor and Head, Department of pediatrics unit 1, First Affiliated Hospital of Jiamusi University. \\ 3. Department of Gynecology and Obstetrics, First Affiliated Hospital of Jiamusi University. \\ 4. School of Stomatology, Department of Orthodontics and Dentofacial Orthopedics, Second Affiliated Dental \\ Hospital of Jiamusi University.
}

\section{Manuscript Info}

Manuscript History

Received: 15 February 2017

Final Accepted: 17 March 2017

Published: April 2017

Key words:-

EAAT2, Rock2, HIE, Excitotoxicity,

Glutamate, Rock inhibitor.
Abstract

Objective: To evaluate fasudil effects on EAAT2 and ROCK2 protein expressions in rat model of neonatal hypoxic-ischemic brain damage (HIBD).

Methodology: 144 Seven-day-old Wistar rats were randomly divided into sham, HIBD, and fasudil groups. Fasudil group were injected intraperitoneally with that compound. Rat brains at 2, 6,12, 24, 48 and $72 \mathrm{~h}$ after HIBD were collected to determine histopathological damage and the expression levels of EAAT2 and ROCK2 proteins selected as the average optical density (AOD). Results were reported as average $(\bar{x}) \pm$ standard deviation (SD), and single-factor analysis of variance (ANOVA) was performed between the multiple groups. A value of $\mathrm{P}$ $<0.05$ was considered statistically significant.

Results: Histopathological damage was reduced in fasudil groups compared with the untreated HIBD group. The expression of ROCK2 in the HIBD group was significantly higher than that in SHAM and fasudil groups at the same times. EAAT2 expression was significantly decreased in HIBD group. Fasudil increased that expression.

Conclusion: Fasudil reduced expression of Rock2 and increased expression of EAAT2. The increase in the expression of EAAT2 constitutes one of the pathways by which fasudil acts and that can explain its neuroprotective effects. Nevertheless further investigation should be conducted to better understand the mechanism of action of fasudil in neuroprotection it could bring in this disease.

Copy Right, IJAR, 2017,. All rights reserved.

\section{Introduction:-}

The hypoxic-ischemic encephalopathy (HIE) of newborn secondary to perinatal asphyxia is quite common, it occurs in 2 infants per 1000 live births. It is responsible for a high mortality in the world, estimated at $23 \%$, annual neonatal deaths $[1,2]$. It is also a source of neurodevelopmental sequelaes $(20 \%)$ of cases of cerebral palsy [CP] of the child to term) [2]. 
In developed countries, perinatal asphyxia (lack of oxygen in the brain at the time of birth) occurs in 3-5 infants per 1000 live births and 0, 5-2 infants per 1000 live births develop cerebral lesions in the form of hypoxic-ischemic encephalopathy (HIE)[3]. Nearly 60\% of neonates with HIE die and $25 \%$ of survivors develop significant disabilities[4].In less affluent countries, the incidence is reported to be higher; 26 per 1000[5].

Glutamate plays a pivotal role in the pathophysiology of HIE, glutamate which is the major excitatory neurotransmitter in the mammalian brain, acting on ionotropic (AMPAr, KAr and NMDAr) and metabotropic (mGluRs) receptors. The ionotropic receptors produce fast-acting excitatory effects, while mGluRs (G proteincoupled receptors) have a crucial role in the modulation of glutamatergic neurotransmission.

The balance of glutamate in the CNS is assured mainly by the excitatory amino-acid transporters and Rock plays a role in their functioning.

Glutamate transporters play a major role in the maintaining brain homeostasis. Astrocytic EAATs play important roles in various neuropathologies in which astrocytes undergo cytoskeletal changes [6,7].

In addition, Rock plays a major role in determining the surface expression of EAAT1/2, providing evidence for an association between transporter function and astrocytic glutamate uptake. Rock inhibitors elevate glutamate transporter function; this activity profile may contribute to their beneficial effects on neuropathologies [8].

Fasudil hydrochloride (Hexahydro-1-(5-isoquinolinyl sulfonyl)-1-H-1, 4-diazepine mono hydrochloride, also known as HA 1077), a new type of isoquinoline sulfonylamide derivatives, is at present only used in clinic as selective inhibitor of Rho kinase for preventing or improving the cerebral vasospasm after subarachnoid hemorrhage and symptoms of cerebral ischemia. However, recent studies found that it can promote survival of neuronal stem cells, axonal regeneration and differentiation of bone marrow mesenchymal cell into neurons $[9,10]$.

Studies about Fasudil and neonatal hypoxic-ischemic encephalopathy, in animal model have been found, which highlighted its anti-inflammatory, anti-apoptosis effects, and so on [11].

It remains unclear whether changes in the Rho signaling pathway affect EAATs expression, following neonatal hypoxic-ischemic encephalopathy.

Thus, the present study aims to evaluate the effects of the Rho kinase inhibitor (fasudil hydrochloride), on changes in Rock, EAAT2 expressions in a rat model of neonatal hypoxic-ischemic encephalopathy injury.

\section{Material and Method:-}

This study is an experimental study on the rat model, conducted in the laboratory of Jiamusi University.

\section{Material:-}

Subjects:-

A total of 144 healthy Wistar rats aged 7 days were purchased from the Jiamusi University Laboratory Animal Center, weighing 10 23 g, male and female open. They were randomly divided into 3 groups: 1) the sham group: intraperitoneally injected with normal saline; 2) HIBD group: intraperitoneally injected with normal saline; 3 ) fasudil group: intraperitoneally injected with fasudil at a dose of $10 \mathrm{mg} \cdot \mathrm{kg}-1 \cdot$ day-1

In addition, rats of each group were randomly divided into 6 subgroups by observation time of 2, 6, 12, 24, 48 and $72 \mathrm{~h}$. All rats of the HIBD and fasudil groups were performed strictly according to the Rice procedure. All experiments conformed to the Guidelines for Animal Experimentation at School of Medicine, Jiamusi University, on the ethical use of animals, and all efforts were made to minimize the number of animals used and their suffering.

Drugs and reagents

- Fasudil injection: Tianjin Red Sun Pharmaceutical Co., Ltd.

- Rabbit anti-mouse Rock2 (polyclonal) antibody : Abcam, Cambridge, UK.

- $\quad$ EAAT2 polyclonal antibody: Santa Cruz Co.

- Rabbit two-step immunohistochemistry kit: Beijing Zhongshan Golden Bridge Company

- Goat serum: Beijing Zhongshan Golden Bridge Company 
- 4\% paraformaldehyde: Virgin biotechnology company

- PBS solution: Beijing Zhongshan Golden Bridge Company

- DAB kit: Beijing Zhongshan Golden Bridge Company

- An anti-dilution: Beijing Zhongshan Golden Bridge Company

- Coverslips: Beijing Zhongshan Golden Bridge Company

- Lysine anti-off slides: Beijing Zhongshan Golden Bridge Company

- An anti-dilution: Beijing Zhongshan Golden Bridge Company

\section{Experimental Equipment:-}

Conventional surgical instruments, paraffin slicing machine, paraffin embedding machine, electronic balance, digital microscope camera systems, bio-optical microscope, refrigerator, ultra-low temperature freezer, constant temperature water bath and bake sheet, high-speed low-temperature centrifuges, medical microwave, fluorescence spectrophotometer instrument, Image Pro Plus-6.0 image analysis software.

\section{Methods:-}

Neonatal rat model of hypoxic-ischemic encephalopathy and treatment design

Briefly, a less than $1 \mathrm{~cm}$ longitudinal midline incision of the neck was performed under ether anesthesia on P7 rats. The right carotid artery was exposed and permanently ligated with 4-0 surgical silk. The surgery lasted less than 5 min. Animals with excessive bleeding were excluded. The rat pups were returned to home cage with their dam for 1 $\mathrm{hr}$ followed by exposure to hypoxia $\left(92 \% \mathrm{~N}_{2}+8 \% \mathrm{O}_{2}\right)$ for $2 \mathrm{hrs}$ by placing them in an airtight chamber partially submersed in a $37^{\circ} \mathrm{C}$ water bath. At the end of $2 \mathrm{hrs}$ hypoxia, the pups were returned to their dam again for recovery.

\section{Drug Injection:-}

Rats in the fasudil hydrochloride group were intraperitoneally injected with $10 \mathrm{mg} / \mathrm{kg}$ fasudil hydrochloride less than 6 hours after hypoxia-ischemia, once by day. Rats in the saline group were intraperitoneally injected with an equal volume of saline, once by day.

Rats in the hypoxia-ischemia group and sham operation group were conventionally fed as Rats in fasudil hydrochloride group. 8 rats were sacrificed in each group at various time points.

\section{HE immunohistochemical staining and specimen collection and slice production:-}

Each subgroup randomly selected eight mice; mice were anesthetized with ether, quickly open the chest, with a 20 $\mathrm{mL}$ syringe infusion of saline in the left ventricle, but cut the right atrial appendage, to the outflow of liquid to clear up. Reperfusion $4 \%$ paraformaldehyde, $0.1 \mathrm{~mol} / \mathrm{L} \mathrm{PBS} 100 \mathrm{~mL}$, to the liver and lungs obviously clear white. After whole brain edema will be decapitated, and immediately save it in $4 \%$ paraformaldehyde, $0.1 \mathrm{~mol} / \mathrm{L}$ PBS solution, fixed $72 \mathrm{~h}$ or more. The fixed brain tissue from mouse brain do after $2 \sim 6 \mathrm{~mm}$ at the optic chiasm to $5 \mu \mathrm{m}$ thickness coronal serial sections of brain tissue blocks of graded alcohol dehydration after taking the optic chiasm, xylene underwent embedded in paraffin, sliced mounted on slides on standby.

\section{Histopathological HE staining:-}

Each group of mice brain tissue sections were routine hematoxylin - eosin (hematoxylin-eosin, HE) staining Color, morphology observed in slices of brain tissue under an optical microscope, inflammatory cell infiltration, inflammatory lesions of the site conditions.

\section{Immunohistochemical expression assay Rock2, EAAT2 in brain tissue:-}

Conventional procedures were used with some modifications. Briefly, brain slices are rehydrated with decreasing concentrations of ethanol $(100 \%, 95 \%, 75 \%, 50 \%)$ for 5 minutes each and washed with phosphate buffered saline (PBS) .The background staining was neutralized (blocked) by using protein block. After washing with PBS, the slices were incubated with the primary antibodies by the following dilution ratios: Anti-MBP: 1: 200, sc-13914, Santa Cruz biotechnology Inc., Santa Cruz, CA), Anti-EAAT 2: 1: 100, \# 3838s, Cell Signaling Technology, Danvers, MA), rabbit anti-Rock2 polyclonal antibody : Abcam, Cambridge, UK, with a working concentration of $1: 200$ at $4^{\circ} \mathrm{C}$ for 18 hours.

The sections were processed for 2 hours at room temperature with horseradish peroxidase -conjugates secondary antibody (1: 1000, sc-2352, Santa Cruz) for MBP (Myelin Basic Protein), or incubated with Novo-Link TM polymer 
for 30 minutes for EAAT2 and ROCK2. Substrate 3, 3'-Diamino benzidine (DAB, Dako, Denmark) was added for less than 5 minutes. Slides were examined with a computer- assisted Olympus BX51 microscope and images were taken with the image-pro- Plus6.0 software analysis system measurement.

\section{Results:-}

Histological change with HE staining:-

The right hemisphere showed evidence of cellular shrinkage and darkening, extending throughout the ischemic cortex following HIBD. Injured neurons and cell loss were noted in both the hippocampus and cerebral cortex. Conventional stains such as HE showed cerebral edema, eosinophilic staining, homogeneous change, and shrinkage in neurons under the light microscope. Moderate edema of cells and diffuse neuronal degeneration were the most abundant changes in the HIBD group at 3 days. Similar changes were much reduced in the fasudil group. In fasudil group, however, numerous damaged neurons were observed as compared to the sham group. There was no neuronal damage or edema in the Sham group.

Rock2 protein expression by immunohistochemical staining after HIBD at different time points.

Table1 has shown Rock2 protein expression (as average optical density, AOD) by immunohistochemical staining after HIBD damage at different time points .

By immunohistochemical staining, we observed the changes of ROCK2 protein expression at different time points after HIE. The expression level of ROCK2 protein increased $6 \mathrm{~h}$ after HIBD and the increase was significant compared with the sham and fasudil groups $(P<0.05)$ at different points of time.

Table 1:- Rock2 protein expression (AOD ( $\overline{\mathrm{x}} \pm \mathrm{SD}), \mathrm{n}=8$ ) by immunohistochemical staining after HIBD damage.

\begin{tabular}{|l|l|l|l|}
\hline GROUP & SHAM & HIBD & FASUDIL \\
\hline $2 \mathrm{~h}$ & $0.694 \pm 0.012$ & $1.093 \pm 0.030^{*}$ & $0.944 \pm 0.013^{* \#}$ \\
\hline $6 \mathrm{~h}$ & $0.702 \pm 0.011$ & $1.372 \pm 0.017^{*}$ & $0.883 \pm 0.012^{* \#}$ \\
\hline $12 \mathrm{~h}$ & $0.698 \pm 0.016$ & $1.201 \pm 0.019^{*}$ & $0.855 \pm 0.007^{* \#}$ \\
\hline $24 \mathrm{~h}$ & $0.701 \pm 0.017$ & $1.158 \pm 0.011^{*}$ & $0.792 \pm 0.006^{* \#}$ \\
\hline $48 \mathrm{~h}$ & $0.699 \pm 0.008$ & $1.056 \pm 0.010^{*}$ & $0.753 \pm 0.011^{* \#}$ \\
\hline $72 \mathrm{~h}$ & $0.692 \pm 0.008$ & $0.957 \pm 0.007^{*}$ & $0.750 \pm 0.007^{* \#}$ \\
\hline
\end{tabular}

Note: * $\mathrm{P}<0.05$ compared with Sham group; ${ }^{\#} \mathrm{P}<0.05$ compared with HIBD group.

EAAT2 protein expression by immunohistochemical staining after HIBD

Table2 indicates expression of EAAT2 protein (AOD $(\bar{x} \pm S D), n=8)$ in brain tissue (cerebral cortex and hippocampus) by immunohistochemical staining after HIBD at different time points.

EAAT2 protein expression was observed in glial cells and neurons in newborn rat brain tissue (cerebral cortex and hippocampus). The expression of EAAT2 protein in the HIBD group decreased significantly with time and was much lower than in Sham at same point in time $\mathrm{P}<0.01$ and in fasudil groups (table2). In the fasudil group, the expression of EAAT2 protein decreased at 2 and $6 \mathrm{~h}$, and then increased at $12 \mathrm{~h}, 24 \mathrm{~h}, 48 \mathrm{~h}$ and 3 days. There was a significant an increase between $6 \mathrm{~h}$ and 48 days $(\mathrm{P}<0.01)$.

Table 2:- EAAT2 protein expression (AOD ( $\overline{\mathrm{x}} \pm \mathrm{SD}), \mathrm{n}=8)$ by immunohistochemical stain after HIBD at different points in time.

\begin{tabular}{|lccc|}
\hline GROUP & SHAM & HIBD & FASUDIL \\
\hline $2 \mathrm{H}$ & $0.1794 \pm 0.0142$ & $0.1398 \pm 0.0165^{*}$ & $0.1387 \pm 0.0138^{*}$ \\
$6 \mathrm{H}$ & $0.1768 \pm 0.0134$ & $0.1305 \pm 0.0112^{*}$ & $0.1317 \pm 0.0193^{*}$ \\
$12 \mathrm{H}$ & $0.1787 \pm 0.0125$ & $0.1106 \pm 0.0175^{*}$ & $0.1425 \pm 0.0149^{*}$ \\
$24 \mathrm{H}$ & $0.1805 \pm 0.0187$ & $0.1068 \pm 0.0160^{*}$ & $0.1537 \pm 0.0169^{*} \#$ \\
$48 \mathrm{H}$ & $0.1834 \pm 0.0162$ & $0.1028 \pm 0.0169^{*}$ & $0.1680 \pm 0.0156^{* \#}$ \\
$72 \mathrm{H}$ & $0.1857 \pm 0.0155$ & $0.0978 \pm 0.0163^{*}$ & $0.1710 \pm 0.0149^{*} \#$ \\
\hline
\end{tabular}

Note: * $\mathrm{P}<0.05$ compared with Sham group; \# $\mathrm{P}<0.05$ compared with HIBD group. 


\section{Discussion:-}

In a rat model of neonatal HIE, altered expression of glutamate transporter and decreased GLT1 expression were observed in the area of ischemic core. Prolonged hypoxia reduced GLT1 expression in astrocytes resulting in extracellular glutamate accumulation [12]. Moreover, functional reversal of glutamate transporter in glial cells occurred during hypoxia and ischemia also contributed to the toxicity of excessive extracellular glutamate. Those researches on EAATs were done with antibiotics, especially, the third generation of cephalosporins, as ceftriaxone [12].

In this study, we used an approved Rho kinase inhibitor in treatment of subarachnoid hemorrhage induced spasm, fasudil. Using immunohistochemistry, we have found that fasudil increased expression of EAAT2 protein in rat model of neonatal hypoxic-ischemic encephalopathy. As mentioned above, this study aimed to observe the influence of fasudil on the expressions of the EAAT2 and Rock2 proteins in neonatal hypoxic-ischemic encephalopathy. This is the first study conducted in this direction. Nevertheless, several studies in vitro and in vivo have shown the effect of fasudil on the expressions of Rock 2 and EAAT2 and even the mechanism that can explain this effect.

This study showed with immunohistochemical analysis that the expression of Rock 2 increased in hypoxic-ischemic encephalopathy while expression of EAAT2 decreased. At the same time, administration of fasudil, which is an inhibitor of Rock2, decreased the expression of Rock 2 and increased the expression of EAAT2. This increase of EAAT2 expression was more significant after 2 days of administration of fasudil. These findings are consistent with those observed by several studies that moreover, have given further details[10,12].

In the CNS the majority of synapses are in close apposition with astrocytes, and astrocytic EAATs appear to be responsible in great part, of Glutamate transport in the CNS[12]. Numerous in vitro and in vivo studies have shown mechanisms by with fasudil acts on EAATs expression. Beart PM et al. demonstrated that transgenic ablation, antisense down-regulation or astrocytic EAATs pharmacological inhibition result in increased extracellular Glutamate and neuronal death. Furthermore, investigations of the distribution and function of different EAAT subtypes confirm the functional dominance of the astrocytic transporters [12].

Other authors found that Astrocytic EAATs also serve another vital role in the CNS, where they provide Glutamate for metabolic processes including the glutamate-glutamine cycle[13]. Thus, a body of evidence suggests that alterations in Glutamate metabolism or astrocytic function resulting from impaired Glutamate transport may also contribute to neuronal damage[14]. As well as reducing the potentially toxic build-up of extracellular Glutamate, it is likely that Glutamate uptake by glial EAATs also signals the energy needs of nearby neurons via activation of the $\mathrm{Na}+\mathrm{K}+$ ATPase and glucose transporters, and changes in the levels of ATP and lactate [15]. Given that activity of EAAT contributes to the determinant role of astrocytes in synaptic function regulation and the increasing evidence for an inter-dependency between astrocytic phenotype and EAAT function, Lau CL et al.[10] found a role not yet determined for the ROCK/Rho system in this relationship. Their findings were consistent with the observations of Abe $\mathrm{K}$ et al. of stellation of cultured astrocytes after inhibition of Rho kinase by Fasudil and Y-27632[16]. They have found that Fasudil induced a shift in the F19 G-actin ratio towards a predominance of monomeric G-actin, indicating that actin was central to the cytoskeletal changes, and that there was a sensible increasing of both Glutamate transport and the cell surface expression of EAAT1 and EAAT2.

As inhibitors of Rock have emerged as neuro-regenerative drug candidates, probably by minimizing glial scarring[ 17], the finding of their capacity to elevate Glutamate transport demonstrate an additional beneficial action likely to contribute to their therapeutic benefits in brain injury.

\section{Conclusion:-}

Fasudil reduced expression of Rock2 and increased expression of EAAT2.Given the widespread involvement of excitotoxicity in acute and chronic neuropathologies, the action of ROCK inhibitors on EAAT2 protein expression represents a beneficial mechanism likely to contribute to their therapeutic benefits in brain injury. Nevertheless further investigation should be conducted to better understand the mechanism of action of fasudil in neuroprotection it could bring in the neonatal hypoxic-ischemic encephalopathy. 


\section{References:-}

1. Cousens S, Lawn JE, Zupan J. Four Million Neonatal Deaths: When? Where? Why? The Lancet. 2005;365(9462):891-900.

2. Robertson CM, Perlman M. Follow-up of the term infant after hypoxic-ischemic encephalopathy. Paediatrics \& child health. 2006;11(5):278.

3. Levene M, Grindulis H, Sands C, Moore J. Comparison of two methods of predicting outcome in perinatal asphyxia. The Lancet. 1986;327(8472):67-69.

4. Vannucci RC. Current and potentially new management strategies for perinatal hypoxic-ischemic encephalopathy. Pediatrics. 1990;85(6):961-968.

5. Cotten CM, Shankaran S. Hypothermia for hypoxic-ischemic encephalopathy. Expert review of obstetrics \& gynecology. 2010;5(2):227-239.

6. Kucukdereli H, Allen NJ, Lee AT, et al. Control of excitatory CNS synaptogenesis by astrocyte-secreted proteins Hevin and SPARC. Proceedings of the National Academy of Sciences. 2011;108(32):E440-E449.

7. Lau CL, Beart PM, O'Shea RD. Transportable and non-transportable inhibitors of L-glutamate uptake produce astrocytic stellation and increase EAAT2 cell surface expression. Neurochemical research. 2010;35(5):735-742.

8. Gerald F. Combs, Jr. The vitamins fundamental aspect in nutrition and health, Third Edition, Elsevir Inc., Amsterdam. 2008;143.

9. Zagami CJ, Beart PM, Wallis N, Nagley P, O'shea RD. Oxidative and excitotoxic insults exert differential effects on spinal motoneurons and astrocytic glutamate transporters: Implications for the role of astrogliosis in amyotrophic lateral sclerosis. Glia. 2009;57(2):119-135.

10. Lau C, O'shea R, Broberg B, Bischof L, Beart P. The Rho kinase inhibitor Fasudil up-regulates astrocytic glutamate transport subsequent to actin remodelling in murine cultured astrocytes. British journal of pharmacology. 2011;163(3):533-545.

11. Ding J, Li Q-Y, Yu J-Z, et al. Fasudil, a Rho kinase inhibitor, drives mobilization of adult neural stem cells after hypoxia/reoxygenation injury in mice. Molecular and Cellular Neuroscience. 2010;43(2):201-208.

12. Beart P, O'shea R. Transporters for L-glutamate: An update on their molecular pharmacology and pathological involvement. British journal of pharmacology. 2007;150(1):5-17.

13. Hawkins RA, O'kane RL, Simpson IA, Vina JR. Structure of the blood-brain barrier and its role in the transport of amino acids. The Journal of nutrition. 2006;136(1):218S-226S.

14. Ré DB, Boucraut J, Samuel D, Birman S, Goff KL, Had-Aissouni L. Glutamate transport alteration triggers differentiation-state selective oxidative death of cultured astrocytes: a mechanism different from excitotoxicity depending on intracellular GSH contents. Journal of neurochemistry. 2003;85(5):1159-1170.

15. Voutsinos-Porche B, Bonvento G, Tanaka K, et al. Glial glutamate transporters mediate a functional metabolic crosstalk between neurons and astrocytes in the mouse developing cortex. Neuron. 2003;37(2):275-286.

16. Abe K, Misawa M. Astrocyte stellation induced by Rho kinase inhibitors in culture. Developmental brain research. 2003;143(1):99-104.

17. Abd-El-Basset EM, Fedoroff S. Upregulation of F-actin and $\alpha$-actinin in reactive astrocytes. Journal of neuroscience research. 1997;49(5):608-616. 